\title{
Acknowledgment to Reviewers of CIMB in 2021
}

\section{CIMB Editorial Office}

Citation: $C I M B$ Editorial Office.

Acknowledgment to Reviewers of CIMB in 2021. Curr. Issues Mol. Biol. 2022, 44, 640-643. https://doi.org/ $10.3390 / \mathrm{cimb} 44020044$

Published: 26 January 2022

Publisher's Note: MDPI stays neutral with regard to jurisdictional claims in published maps and institutional affiliations.

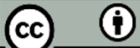

Copyright: (C) 2022 by the author. Licensee MDPI, Basel, Switzerland. This article is an open access article distributed under the terms and conditions of the Creative Commons Attribution (CC BY) license (https://creativecommons.org/licenses/by/4.0/).

MDPI AG, St. Alban-Anlage 66, 4052 Basel, Switzerland

Rigorous peer-reviews are the basis of high-quality academic publishing. Thanks to the great efforts of our reviewers, $C I M B$ was able to maintain its standards for the high quality of its published papers. Thanks to the contribution of our reviewers, in 2021, the median time to first decision was 17 days and the median time to publication was 35 days. The editors would like to extend their gratitude and recognition to the following reviewers for their precious time and dedication, regardless of whether the papers they reviewed were finally published:

Abdullah Nahain

Abhishek Dey

Abhishek Kumar Singh

Achinto Saha

Adalbert Schiller

Aditi Swarup

Adriana López-Dóriga

Agnieszka Karbownik

Agnieszka Markowska

Agnieszka Potęga

Agueda González Rodríguez

Alba Rodríguez-García

Alec McCarthy

Alejandro Madrid

Aleksandar Ž. Kostić

Aleksandra Karczmarska-Wódzka

Alessandra Ferramosca

Alessandro Sinigaglia

Alexander Berezin

Alexandre Buffet

Alexandre Lamas

Alexandros Papachristodoulou

Alfredo Caturano

Alfredo Grilli

Ali R. Jazirehi

Amir Shemirani

Ana Domenech

Ana I Arroba

Andras Bikov

André L. Simão

Andreas Weber

Angelija Valančiūtè

Anil Verma

Anju Kumari
Ankita Srivastava

Anna Lopata

Anna Makuch-Kocka

Anna V. Klepikova

Anne-Sophie Lia

Antonio Simone Laganà

Antony Kam

Arto Pulliainen

Ashutosh Pandey

Astrid Brull

Attayeb Mohsen

Augustin M. Ofiteru

Ayse Kose

Basem Battah

Basil Grüter

Beatriz G. De La Torre

Biserka Relic

Biswarup Saha

Bryan Fuller

Budimir Mijović

Byron Baron

Camilla Urbaniak

Carlo Pozzi

Carlos Perez-Ramirez

Carmela Paolillo

Carmela Saturnino

Chao lien Liu

Charalampos Siristatidis

Charanya Kumar

Chia-Hung Chou

Chichun Ho

Chip Hahn

Chung-Jung Liu

ChunNian $\mathrm{He}$ 
Cinzia Bertea

Cinzia Giacometti

Clivel Charlton

Cristina Maccallini

Cyril Eleftheriou

D. A. Crosby

Daniel Martin

Daniela Rosado

Danilo Licastro

Dariusz Kucharczyk

David Stegner

David Zaitlin

Davide Barreca

Deborah Galpert

Demin Cai

Dilip Shah

Dimitra Micha

Dolors Puigoriol

Drago Beslo

Dustin J. Hines

E Scott Sills

Elena Canciani

Elena López-Jiménez

Elena Rapizzi

Elena Sommariva

Eliana Dell'olmo

Elisha Otieno Gogo

Eva Bagyinszky

Eva Bazsalovicsová

Eva Tvrda

Evgenia Halkia

Eylem Kulkoyluoglu Cotul

Fabio Cavaliere

Farid Chemat

Felipe Martinez-Pastor

Fernando Cardona

Francesco Bianco

Francesco Cadario

Francesco Ferrini

Francisca Rodrigues

Francisco Centeno

Francisco Epelde

Francisco Ferreira-da-Silva

Francisco Guillen-Grima

Gabriel Santpere

George Sangster

Georgeta Violeta Trusca

Georgia Ntali

Gerd Bungartz

Gergana Deevska

Giuseppe Mannino
Grzegorz Trybek

Guangde Jiang

Guannan Wang

Guendalina Zuccari

Hae-Sang Lee

Haoshen Shi

Harada Kouji

Haridha Shivram

Hesham R. Hesham R. El-Seedi

Himangshu Sonowal

Hiroaki Okae

Ho Jeong Lee

Hsueh-Wei Chang

Hubert Sytykiewicz

Hui-Hua Hsiao

Hung-Pin Tu

Ilaria Dettori

Ioannis Liampas

Ivan Salazar

Ivana Škrlec

J. Catharina Duvigneau

Jack Rogers

James A. Hendrix

Janusz Strychalski

Jason E. Swain

Javier Rodríguez Villanueva

Jayadev Mavuluri

Jayant S Vaidya

Jean Boutin

Jianfeng Huang

Jiarui Bi

Jingquan Wang

Joana Caetano

Joanna Wierońska

João M. Santos

José A. Lupiáñez

José Andrés Yunes

Jose Antonio Roldán-Nofuentes

Joseph O. Falkinham III

Josko Bozic

Józef W. Kobos

Juan Ramón Tejedor

Juei-Tang Cheng

Julie Rageul

Jun-Beom Park

Kamil Grubczak

Kandi Sridhar

Kang Sup Kim

Katalin Sipos

Katarina Sebekova

Kathryn Y. Burge 
Katsuhiko Ishihara

Kavita Sharma

Kay Ramsay

Kazutoshi Nishijima

Kei Maruyama

Kendrick Koo

Kiyoshi Takagi

Kok-Min Seow

Konstantin M.J. Sparrer

Kranjc Simona

Kristina Kuhbandner

Krutika Patel

Krzysztof Waleron

Kuboyama Tomoharu

Kwang-Hoon Chun

Kyojiro N. Ikeda

Larisa Anghel

Laura Lõpez-Pingarrõn

Leonardo De Assis

Letizia Palomba

Liqing Zang

Lisha Liu

Lola Rueda-Ruzafa

Lorena Tedeschi

Luana Beatriz Dos Santos Nascimento

Lubka T. Roumenina

Luca Gallelli

Luca Testarelli

Luigi Bennardo

Luís Pedro Ferreira Rato

Łukasz Szczukowski

Łukasz Zapała

Magdalena Jeszka-Skowron

Mahesh Vishe

Mahmoud A.O. Dawood

Malini Mukherjee

Manos Vlasiou

Manu Kumar

Marco Tafani

Margret H. Bülow

Maria Susana Lopes

Mária Szekeres

Mariam Gaid

Marian Price-Carter

Maribel Forero-Castro

Marina Piscopo

Marjan Rupnik

Markus Schirmer

Marta Gomarasca

Martin Kolisek

Martin Pešl
Masaki Okamoto

Massimo Venditti

Maurizio Forte

Mayuren Candasamy

Md. Moshfekus Saleh-E-In

Michal Alexovič

Miguel Prieto

Mihai Cornel Traian Dimitriu

Milica Nedeljković

Miriam Hickey

Mirosław Ratkiewicz

Mithun Mitra

Mohan Kumar Muthu Karuppan

Mona Batish

Nagarajarao Shamaladevi

Nanako Kawaguchi

Nancy N. Nichols

Natalia Maria Serwin

Nicholas Vrettos

Nikolaj Travica

Nikolaos Nikoloudakis

Norihiko Ohbayashi

Olivier Malaise

Paolo Martelletti

Paško Konjevoda

Patricia Ondine Lucaciu

Patricio Iturriaga-Vasquez

Peng Qi

Peng Zhou

Péter Csécsei

Peter E. Murray

Peter Guttmann

Peter Jones

Philip J. Young

Philippe B. Wilson

Philippe Colin

Phillipp Brockmeyer

Photini Mylona

Piero Cossu

Pietro Napoli

Pradeep Singh

Radek Vodicka

Radu Liviu Sumalan

Rafael Franco

Raghavendran Partha

Rahul Mallick

Raja Veerapandian

Rajiv Doddamani

Rajkumar Sahani

Ram Vinay Pandey

Rebecca Berrens 
Renin Chang

Reza Shafiei

Rita Benítez

Robert L. Jarret

Roberto Cannataro

Rodrigo A Loiola

Rongkung Tsai

Rouba Nasreddine

Sabata Pierno

Samaila Musa Chiroma

Samuel Long

Sandip Ashok Sonar

Sanjit Mukherjee

Sara R. Martins-Neves

Saranya Canchi

Sascha Schäuble

Saskia Trump

Saurabh Chaudhary

Seiji Miyauchi

Sekyung Oh

Sergio Occhipinti

Seung-youn Jung

Shah Adil Ishtiyaq Ahmad

Shauna Kaplan

Sheyla González

Shin Toyoda

Shugo Mizuno

Sigrid Müller-Deubert

Silvia Liskova

Silvia Martu

Sivaraman Natarajan

Sophia Letsiou

Soumya Poddar

Sree V. Chintapalli

Srihari Konduri

Stefan Kabisch

Subhadip Choudhuri
Sunil Karhadkar

Takaaki Senbonmatsu

Takayuki Kawato

Talha Bin Emran

Tatiana Wojciechowicz

Tatyana V. Savchenko

Teresa Grzelak

Thomas Schmutzer

Tom Huxford

Tomasz Wenta

Toshitsugu Fujita

Tsvetelina Velikova

Urszula Jankiewicz

Vangelis D. Karalis

Vasilios Tsarouhas

Veronique M. Braud

Vijaya Kumar Narne

Viktória Fisi

Vishruti Makani

Vlad Pădureanu

Walther Bild

Wataru Nakata

$\mathrm{Xi}$ Chen

Xiaoxiao Huang

Xin Chen

Yafeng Ma

Yali Sun

Yang Jinrui

Yi-Chiung Hsu

Yoon Jung Park

Young-Kwon Seo

Yuemin Bian

Zbigniew J. Witczak

Zehuan Liao

Zhenlin Han

Zhongwei Zou

Zhuqiu Jin 